\title{
Effects of a mandatory DRG payment system in South Korea: Analysis of multi- year nationwide hospital claims data
}

\author{
Jae Woo Choi ${ }^{1,2}$, Seung-Ju Kim², ${ }^{2,3}$ Hye-Ki Park ${ }^{2,4}$, Sung-In Jang ${ }^{2,5}$, Tae Hyun Kim ${ }^{2,6}$ and Eun-Cheol Park ${ }^{2,5,6^{*}}$ (D)
}

\begin{abstract}
Background: In 2002, a voluntary diagnosis-related groups (DRGs) payment system was introduced in South Korea for seven disease groups, and participation in the DRGs was mandated for all hospitals beginning in 2013. The primary aim of this study was to compare results reflective of patient care between voluntary participation hospitals (VPHs) and mandatory participation hospitals (MPHs) governed by either the DRGs or fee-for-service (FFS) payment system.

Methods: We collected DRGs and FFS inpatient records $(n=3,038,006)$ from the Health Insurance Review and Assessment for the period of July 2011 to July 2014 and compared length-of-stay, total medical costs, shifting services to an outpatient setting, and readmission rates according to payment system, time of DRGs implementation, and hospital type. We analyzed the effects of mandatory introduction in DRGs payment system on results for patient care and used generalized estimating equations with difference-in-difference methodology.

Results: Most notably, patients at MPHs had significantly shorter LOS and lower readmission rates than VPH patients after mandatory introduction of the DRGs. Shifting services to an outpatient setting was similar between the groups.

Conclusions: Our findings suggest that the DRGs payment policy in Korea has decreased LOS and readmission rates. These findings support the continued implementation and enlargement of the DRGs payment system for other diseases in South Korea, given its potential for curbing unnecessary resource usage encouraged by FFS. If the Korean government deliberates on expansion of the DRGs to include other diseases with higher rates of complications, policymakers need to monitor deterioration of health care quality caused by fixed pricing.
\end{abstract}

Key words: Mandatory DRG policy, Effect evaluation, Difference-in-difference methodology

\section{Background}

Diagnosis-related groups (DRGs) refer to groups of similar patients for consumption patterns of resources and clinical characteristics. DRGs represent a flat per discharge payment that differs based on severity, procedures, and diagnosis [1]. The DRG payment system, as a policy tool for efficiency improvement and cost containment, shifts economic responsibilities from insurers to medical institutions and promotes consciousness for

\footnotetext{
*Correspondence: ECPARK@yuhs.ac

${ }^{2}$ Institute of Health Services Research, Yonsei University College of Medicine,

50-1 Yonsei-ro, Seodaemun-gu, Seoul, Korea

${ }^{5}$ Department of Preventive Medicine, Yonsei University College of Medicine,

50-1 Yonsei-ro, Seodaemun-gu, Seoul, Korea

Full list of author information is available at the end of the article
}

costs among service providers. Thus, medical institutions tend to regulate the payment mechanism by altering medical services [2]. DRG payment systems were first developed at Yale University as an alternative to reimbursement payment system to manage medical expenses. The US government decided to implement to the Medicare program [3, 4]. Numerous Europe countries have also introduced the DRGs payment system to manage medical expenditures and improve efficiency [5].

South Korea started to introduce national health insurance in 1977 and adopted a fee-for service (FFS) system for health care services [6]. FFS refers to a payment system that healthcare provider is paid for each service conducted, and research has indicated that FFS systems

(C) The Author(s). 2019 Open Access This article is distributed under the terms of the Creative Commons Attribution 4.0 International License (http://creativecommons.org/licenses/by/4.0/), which permits unrestricted use, distribution, and 
result in rapid increases in medical costs [7]. The Korean government decided to introduce a DRGs payment system to solve problems stemming from overtreatment under the FFS system. The Korean government preliminarily adopted a pilot program of the DRGs payment system that reflected national average treatment charges among patients in particular disease groups from 1997 to 2002. Following the pilot stage, Korea implemented a voluntary DRGs payment system for groups of seven disease and gained participation from about $61 \%$ of all hospitals nationwide [8]. As of 2012, the government executed mandatory participation in the DRGs system, beginning with relatively small medical institutions (clinics or hospitals), followed by larger hospitals (general hospitals or tertiary hospitals) in 2013 [9].

For South Korea, previous literatures have studied the effect of implementation of the DRGs policy and have often identified significant reductions in length-of-stay (LOS) and out-of-pocket payments, compared to the FFS system, despite some results to the contrary [10-15]. The effect of the DRGs system for quality of care has also been controversial $[8,16]$. However, these inconsistencies may be explained by the fact that many studies only examined effects in voluntary participation hospitals (VPHs), which likely manage more efficiently than other hospitals. Little is known about the effect of the DRGs payment system utilizing nationwide data from both voluntary and mandatory participation hospitals (MPHs).

Therefore, we examined LOS, total medical cost, shifting services to outpatient settings, and readmission rate for patients from VPHs and MPHs governed by either the DRGs or FFS. We also analyzed the effects of the DRGs policy according to hospital type and date of DRGs implementation.

\section{Methods}

\section{Data sources and study design}

The Health Insurance Review and Assessment (HIRA) dataset includes information on the medical and pharmacy bills for all Koreans. All hospitals or pharmacies turn in claims dataset for outpatient and inpatient care, including demographic information, procedures, diagnoses, and prescriptions, to the HIRA to get reimbursement of medical expenses from the government. The HIRA data were based on the sixth edition of the Korean Classification of Disease, which is a revised version of the tenth edition of the International Classification of Diseases code [17].

From the database, we extracted inpatient DRGs data, because DRGs only apply to admitted patients. We also excluded records from inpatients who received medical aid, as the DRGs system only includes recipients of national health insurance. We merged the DRGs data with another hospital-based dataset related to patients treated under the FFS system: the dataset was extracted by the HIRA using unique patient IDs. We then collected all information of inpatients from July 2011 to July 2014 with one of seven DRG-approved diseases (KDRGs codes: C051, C052, C053, C054 [cataract surgery], D111 [tonsillectomy and adenoidectomy], G081, G082, G083, G084 [appendectomy], G095, G096, G097, G098 [herniotomy], G102, G104, G015, G106 [hemorrhoidectomy], N041, N042, N045, N046, N047, N048 [hysterectomy], and O016, O017 [cesarean delivery and hysterectomy and adneelomy]). In addition, we included general information about the admitting hospital, such as ownership, type, teaching status, location, and numbers of doctors, nurses, and beds, in our dataset. This study received ethical approval for this research from the institutional review board of the Yonsei University Graduate School of Public Health.

\section{Study sample}

We included records of DRGs claim data and FFS claim data and grouped them according to the date DRGs was implemented. Hospitals were classified as clinics; hospitals providing primary care, including inpatient services; general hospitals; and tertiary hospitals with more than 100 beds $[18,19]$. In multivariate analysis, we combined hospital types as large hospitals (general hospitals + tertiary hospitals) and small hospitals (clinics + hospitals).

\section{Dependent variables}

This study explored four items reflective of patient care (LOS, total medical costs, shifting services to outpatient settings, and readmission rates). These items have often been utilized in studies of DRG effects in Korea [15, 20-23]. We assessed LOS using admission date and discharge date. This research estimated total medical costs as the sum of FFS or DRGs claims for each inpatients, and we utilized each year's increasing rate of negotiated medical price to adjust medical expenses to 2014 levels. Shifting services to an outpatient setting was defined as visiting outpatient institutions within 14 days before or after hospitalization based on the admission date or discharge date, respectively. We defined readmission rate as readmission with same primary diagnosis within 30 days after discharge at the same or another hospital. This study excluded results for cataract surgeries in the readmission analysis because hospitals claimed the same disease code when a doctor operated on the patient's other eye before mandatory participation of the DRGs system. This type of readmission differed from our definition, which depended on the readmission being clinically related to a prior admission [24]. 


\section{Independent variables}

This study compared the results on patient care in MPHs and VPHs. An MPH was defined as a hospital that continued with the FFS system until July 2012 (clinics and hospitals) or July 2013 (general and tertiary hospitals), whereas VPHs comprised hospitals that implemented the DRGs system at least 6 months before the mandatory participation of the DRGs system. Hospitals that moved to DRGs payment within 6 months before mandatory participation of the DRGs system were excluded in this study. Thus, the experimental group comprised patients who received care at MPHs, and the comparison group included patients treated at VPHs (Fig. 1).

\section{Covariates}

We matched patient level data to the hospital information at which the patient had been admitted. Patient level data included sex (female, male), age group (20-34, 35-49, 5064, 65-74, 75+ years), and Charlson comorbidity index (CCI; $0,1,2,3,4+$ ), which is constructed based on ICD-9 codes. The Charlson comorbidity index is the sum of weighted scores allocated to main health conditions [25].

Hospital-level data included type (clinics, hospitals, general hospital, and tertiary hospital), ownership (private, public), teaching status (teaching, non-teaching), sizes (number of beds), human resources (number of doctors or nurses), and locations (urban or rural).

\section{Statistical analysis}

This study calculated summary statistics by calculating means and standard deviations for and frequencies and percentages using chi-square test and t-test. Chi-square tests of associations were utilized to assess differences in proportions by mandatory participation in the DRGs system.
This research used multivariate models to evaluate the effect of mandatory participation in DRGs on LOS, total medical cost, shifting services to outpatient settings, and readmission. This study adjusted the models for principal diagnosis and patient- and characteristics in hospital-level and then performed generalized estimating equations (GEE). This study also utilized logit-link GEE to assess shifting services to outpatient settings and 30day readmission and identity-link normal-distribution GEE to evaluate log-transformed total medical cost and LOS.

This study utilized a difference-in-differences methodology to compare pre- and post-reform changes between MPHs and VPHs, in which we controlled for baseline differences between the hospital groups. Previous studies have used the difference-in-differences approach to estimate the effect of policy alteration or introduction of new system [26, 27]. The difference-in-differences methodology is a standard policy assessment tool that explores the independent effect of introduction of policy on a case group in comparison with a control group once any policy is implemented. The case group of this study comprised patients admitted to hospitals that mandatorily participated in the DRGs system, while the control group consisted of those who admitted to hospitals that voluntarily participated in the DRGs system. Using data from patients before the mandatory implementation of DRGs, we were also able to control for pre-reform trend differences to ensure that the estimated impacts were not biased due to different baselines for MPHs and VPHs. The DID estimator can be calculated only if in pre-treatment period parallel trend assumption hold. We tested the parallel trend assumption by hospital sizes (clinics and hospital; general and tertiary hospital) and the parallel trend assumption was satisfied (Additional file 1: Figure S1 and S2).

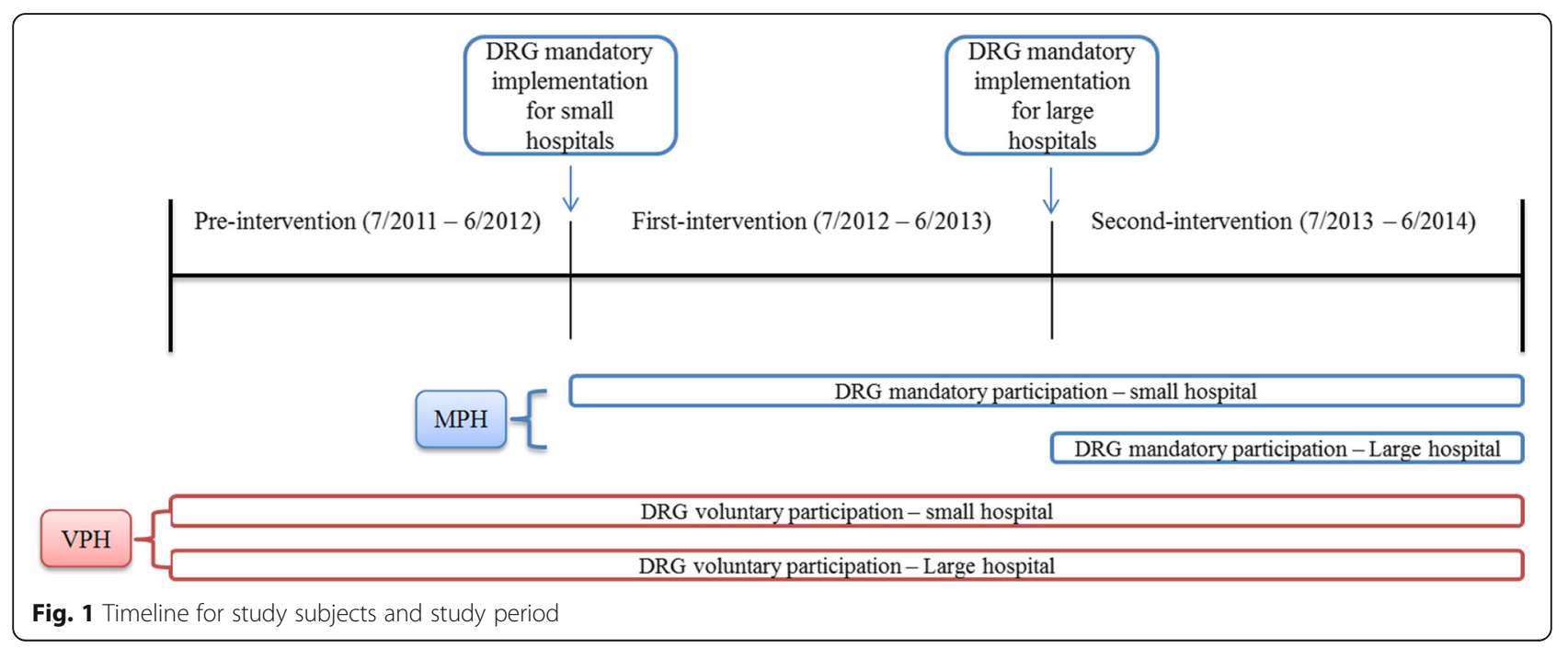




\section{Results}

We collected records from 3,038,006 patients (DRGs claim data: 2,565,902; FFS claim data: 472,104) and grouped them according to date of DRGs policy implementation (pre- intervention: 1,011,057, July 2011 to June 2012; first intervention: 1,014,627, July 2012 to June 2013; or second intervention: 1,012,322, July 2013 to June 2014). We collected records for adults who were discharged with a principal diagnosis for cataract surgery, tonsillectomy and adenoidectomy, appendectomy, herniotomy, hemorrhoidectomy, hysterectomy, or cesarean delivery from one of 3,626 hospitals between July 2011 and July 2014. Of these patients, 28.8\% were cared for by $1,432 \mathrm{MPHs}$, and $71.2 \%$ were cared for by 2,194 VPHs. The mean age of the patients at MPHs was 45.4 years, and $71.4 \%$ were women. Cesarean delivery accounted for $25.7 \%$ of MPH cases, and approximately half of the patients demonstrated a CCI of 0 . The mean age of VPH patients was 54.1 years, and $60.6 \%$ were women. Cataract surgery accounted for $47.5 \%$ of these cases, and $29.1 \%$ of the patients had a CCI of 0 .

Table 1 shows summary statistics of patient and hospital characteristics in this study. At the hospital level, more than half of MPHs were clinics, and approximately $80 \%$ were private medical institutions that depended almost exclusively on payments for their revenue. Over $86 \%$ of MPHs served patients who lived in urban. The average numbers of physicians, nurses, and beds were 29 , 54, and 118, respectively. Approximately $85 \%$ of VPHs were clinics, and $93.6 \%$ were private hospitals. Over $90 \%$ served patients who lived in urban, and the average numbers of VPH physicians, nurses, and beds were 5,9 , and 30 respectively.

Table 2 shows that the average LOS of patients treated at MPHs and VPHs reduced by 1.15 and 0.1 days, respectively, with regard to the first intervention. At the second intervention, LOS for MPH and VPH patients decreased by 0.76 and 0.25 days, respectively. The average total medical cost for $\mathrm{MPH}$ patients increased by $\$ 235$, whereas that for VPH patients decreased by $\$ 27$ after the first intervention. However, the total medical cost for VPH patients increased by $\$ 41$ at large hospitals at the second intervention. Table 3 shows that the shifting of services to outpatient settings among patients cared for at MPHs and VPHs increased by $4.2(\mathrm{p}<.001)$ and $1.1(\mathrm{p}<.001)$ percentage points (p.p.) among small hospitals, respectively (first intervention effect), while this increased by $2.1 \quad(\mathrm{p}<.001)$ and $2.2(\mathrm{p}<.001)$ p.p. among large hospitals (second intervention effect), respectively. Finally, readmission of MPH and VPH patients decreased by 1.1 and 0.1 p.p. among small hospitals (first intervention effect), respectively, and readmission of MPH and VPH patients decreased by 1.2 and 0.3 p.p. among large hospitals (second intervention effect), respectively.
After adjusting for principal diagnosis, patient- and hospital-characteristics, we discovered that the average LOS of MPH patients reduced, compared to VPH patients (adjusted odds ratio: 0.93, 0.89; 95\% confidence interval [CI], 0.92 to $0.94,0.88$ to 0.90 ) (Table 3). We observed these LOS trends for all diagnoses except cataract surgery (Additional file 2: Table S1). The total medical expenses of MPH patients increased (adjusted odds ratio: $1.38,1.15$; $95 \% \mathrm{CI}: 1.37$ to $1.39,1.14$ to 1.16 ) except in cases of tonsillectomy and adenoidectomy and hemorrhoidectomy (Additional file 2: Table S2). Table 3 shows that shifting services to outpatient settings for MPH and VPH patients did not differ significantly (adjusted odds ratio: $0.99,1.00$; $95 \%$ CI: 0.96 to $1.01,0.97$ to 1.03), although we did observe some disease-specific variations (Additional file 2: Table S3). Finally, this study found that readmission rates of MPH patients reduced (adjusted odds ratio: $0.40,0.82$; $95 \%$ CI: 0.36 to 0.45 , 0.70 to 0.96$)$; these trends were similar among all diagnoses (Additional file 2: Table S4).

\section{Discussion}

In our broad observational study, we found that patients treated at MPHs had significantly shorter LOSs and lower readmission rates than VPH patients following mandatory participation of the DRGs and that shifting services to outpatient settings was similar between the patient groups. In addition, the total medical costs incurred by MPH patients increased following mandatory participation of the DRGs system.

First, we found that LOS among MPH patients was significantly lower than that among VPH patients following mandatory participation of the DRGs system. This result was similar to previous findings that indicated the average LOS of patients with certain diagnoses declined during the DRG pilot program (1997 to 2000) and implementation of the voluntary DRG system (2004 to 2011) in South Korea [15, 28]. Results for other countries also indicated that a DRGs payment shortened LOS [2, 29]. One of the major purposes of DRGs systems is to reduce LOS, as well as medical expenditures, for the patient. The average LOS in Korea in 2013 was 16.5 days, indicating it the second highest among the Organization for Economic Cooperation and Development behind Japan (Japan: 17.2 days) [30]. The LOS in Korea is increasing annually, while that in Japan has reduced. It is interested that LOS reduced significantly following DRGs implementation despite the surgical procedures utilized by the Korean DRGs system are simple relatively. More importantly, this significant reduction in LOS may be a long-term effect of the system, as we observed reduced LOS during the second intervention period. Insurers need to monitor treatment processes and 
Table 1 General characteristics of patients and hospitals in this study

\begin{tabular}{|c|c|c|c|c|c|c|c|}
\hline \multirow{2}{*}{\multicolumn{2}{|c|}{ Variables }} & \multirow[t]{2}{*}{ Total } & \multicolumn{2}{|c|}{ Patients in MPHs $(\mathrm{N}: 877,012)$} & \multicolumn{2}{|c|}{ Patients in VPHs $(\mathrm{N}: 2,160,994)$} & \multirow{2}{*}{$\begin{array}{l}p- \\
\text { value }\end{array}$} \\
\hline & & & $\mathrm{N}$ & $\%$ & $\mathrm{~N}$ & $\%$ & \\
\hline \multicolumn{8}{|c|}{ Patient characteristics } \\
\hline \multirow[t]{7}{*}{ Age } & & & & & & & $<.001$ \\
\hline & 20-34 & 771,461 & 308,009 & 35.1 & 463,452 & 21.5 & \\
\hline & $35-49$ & 706,019 & 260,116 & 29.7 & 445,903 & 20.6 & \\
\hline & $50-64$ & 584,997 & 148,509 & 16.9 & 436,488 & 20.2 & \\
\hline & $65-74$ & 585,253 & 97,911 & 11.2 & 487,342 & 22.6 & \\
\hline & $75-$ & 390,276 & 62,467 & 7.1 & 327,809 & 15.2 & \\
\hline & Mean \pm SD - yr & $52.3 \pm 7.1$ & 45.4 & \pm 6.4 & 54.1 & \pm 8.7 & $<.001$ \\
\hline \multirow[t]{3}{*}{ Sex } & & & & & & & $<.001$ \\
\hline & Male & $1,102,541$ & 251,154 & 28.6 & 851,387 & 39.4 & \\
\hline & Female & $1,935,465$ & 625,858 & 71.4 & $1,309,607$ & 60.6 & \\
\hline \multicolumn{2}{|c|}{ Principal diagnosis } & & & & & & $<.001$ \\
\hline & Cataract surgery & $1,191,471$ & 164,350 & 18.7 & $1,027,121$ & 47.5 & \\
\hline & Tonsillectomy \& adenoidectomy & 34,108 & 29,514 & 3.4 & 4,594 & 0.2 & \\
\hline & Appendectomy & 189,782 & 153,249 & 17.5 & 36,533 & 1.7 & \\
\hline & Herniotomy & 62,042 & 40,962 & 4.7 & 21,080 & 1.0 & \\
\hline & Hemorrhoidectomy & 793,650 & 81,239 & 9.3 & 712,411 & 33.0 & \\
\hline & Hysterectomy & 279,737 & 182,766 & 20.8 & 96,971 & 4.5 & \\
\hline & Cesarean delivery & 487,216 & 224,932 & 25.7 & 262,284 & 12.1 & \\
\hline \multicolumn{3}{|c|}{ Charlson comorbidity index (CCl) } & & & & & $<.001$ \\
\hline & 0 & $1,029,110$ & 401,308 & 45.7 & 627,802 & 29.1 & \\
\hline & 1 & 476,180 & 168,393 & 19.2 & 307,787 & 14.2 & \\
\hline & 2 & 377,376 & 99,015 & 11.3 & 278,361 & 12.9 & \\
\hline & 3 & 484,961 & 89,543 & 10.2 & 395,418 & 18.3 & \\
\hline & $\geq 4$ & 670,379 & 118,753 & 13.5 & 551,626 & 25.5 & \\
\hline \multicolumn{8}{|c|}{ Hospital characteristics } \\
\hline \multicolumn{2}{|c|}{ Hospital type } & & & & & & $<.001$ \\
\hline & Tertiary hospital & 44 & 44 & 3.1 & 0 & 0 & \\
\hline & General hospital & 294 & 198 & 13.8 & 96 & 4.4 & \\
\hline & Hospital & 582 & 346 & 24.2 & 236 & 10.8 & \\
\hline & Clinic & 2,706 & 844 & 58.9 & 1,862 & 84.9 & \\
\hline \multicolumn{2}{|c|}{ Hospital ownership } & & & & & & $<.001$ \\
\hline & Public & 36 & 1 & 0.1 & 35 & 1.6 & \\
\hline & Corporation & 405 & 299 & 20.9 & 106 & 4.8 & \\
\hline & Private & 3,185 & 1,132 & 79.1 & 2,053 & 93.6 & \\
\hline \multicolumn{2}{|c|}{ Teaching status } & & & & & & $<.001$ \\
\hline & Teaching & 157 & 122 & 8.5 & 35 & 1.6 & \\
\hline & Non-teaching & 3,469 & 1,310 & 91.5 & 2,159 & 98.4 & \\
\hline \multicolumn{2}{|c|}{ Location } & & & & & & $<.001$ \\
\hline & Urban & 3,216 & 1,237 & 86.4 & 1,979 & 90.2 & \\
\hline & Rural & 410 & 195 & 13.6 & 215 & 9.8 & \\
\hline \multicolumn{2}{|c|}{ Number of bed - mean $\pm S D$} & $148 \pm 106$ & 118 & \pm 86 & 30 & \pm 21 & \\
\hline \multicolumn{2}{|c|}{ Number of doctor - mean $\pm S D$} & $34 \pm 47$ & 29 & \pm 46 & 5 & \pm 4 & \\
\hline \multicolumn{2}{|c|}{ Number of nurse - mean $\pm S D$} & $63 \pm 72$ & 54 & \pm 82 & 9 & \pm 12 & \\
\hline
\end{tabular}

$P$-values were calculated with the use of Chi-square tests (or Student's t-test) of association unless otherwise indicated $\mathrm{MPH}$ mandatory participation hospital

$V P H$ voluntary participation hospital 
Table 2 Regression model estimates for length-of-stay and total medical cost by DRG policy intervention period

\begin{tabular}{|c|c|c|c|c|c|c|c|}
\hline \multirow[t]{3}{*}{ Variables } & \multirow{3}{*}{$\begin{array}{l}\text { Pre-Intervention } \\
7 / 2011-6 / 2012 \\
\text { Mean } \pm \text { SD }\end{array}$} & \multicolumn{3}{|c|}{ First Intervention } & \multicolumn{3}{|c|}{ Second Intervention } \\
\hline & & \multicolumn{3}{|c|}{$7 / 2012-6 / 2013$} & \multicolumn{3}{|c|}{$7 / 2013 \sim 6 / 2014$} \\
\hline & & Mean \pm SD & B & DID & Mean $\pm S D$ & B & DID \\
\hline \multicolumn{8}{|c|}{ Length-of-stay } \\
\hline \multicolumn{8}{|c|}{ Large hospital } \\
\hline $\mathrm{MPH}$ & $5.37 \pm 4.08$ & $5.22 \pm 3.93$ & $0.98^{* * *}$ & 1.01 & $4.21 \pm 2.51$ & $0.86^{* * *}$ & $0.89^{* * *}$ \\
\hline $\mathrm{VPH}$ & $5.71 \pm 3.27$ & $5.52 \pm 2.73$ & $0.97^{* * *}$ & & $5.27 \pm 2.45$ & $0.97^{* * *}$ & \\
\hline \multicolumn{8}{|c|}{ Small hospital } \\
\hline $\mathrm{MPH}$ & $6.49 \pm 2.99$ & $5.34 \pm 2.49$ & $0.91^{* * *}$ & $0.93^{* * *}$ & $4.53 \pm 2.65$ & $0.96^{* * *}$ & $0.97^{* * *}$ \\
\hline VPH & $2.55 \pm 2.18$ & $2.45 \pm 2.06$ & $0.98^{* * *}$ & & $2.35 \pm 2.00$ & $0.99^{* * *}$ & \\
\hline \multicolumn{8}{|c|}{ Total medical cost } \\
\hline \multicolumn{8}{|c|}{ Large hospital } \\
\hline $\mathrm{MPH}$ & $1382 \pm 710$ & $1353 \pm 705$ & $0.98^{* * *}$ & $0.87^{* * *}$ & $1556 \pm 675$ & $1.18^{* * *}$ & $1.15^{* * *}$ \\
\hline VPH & $1370 \pm 497$ & $1549 \pm 568$ & $1.20^{* * *}$ & & $1590 \pm 600$ & $1.02^{* * *}$ & \\
\hline \multicolumn{8}{|c|}{ Small hospital } \\
\hline $\mathrm{MPH}$ & $1032 \pm 401$ & $1267 \pm 440$ & $1.33^{* * *}$ & $1.38^{* * *}$ & $1188 \pm 469$ & $1.01^{* * *}$ & $1.01^{* * *}$ \\
\hline VPH & $836 \pm 216$ & $809 \pm 301$ & $0.95^{* * *}$ & & $802 \pm 309$ & 1.00 & \\
\hline
\end{tabular}

MPH mandatory participation hospitals

$\mathrm{VPH}$ voluntary participation hospitals

DID difference-in-differences

Large hospital: tertiary hospitals + general hospitals; Small hospital: hospitals+ clinics.

Adjusted odds ratios obtained from generalized estimating equations analysis with all of the variables in Table 1.

All costs were controlled by medical insurance fees, which are annually adjusted and include un-insured costs.

1 USD $=1,200$ Korean won $(02 / 2016)$

${ }^{* * *} p<0.001$

results because the DRGs payment system may expedite negative actions, such as inappropriate early discharges of patients who had unstable conditions [31].

Second, total medical costs among MPH patients, but not VPH patients, increased following mandatory participation of the DRGs system, and the magnitude of total medical costs reimbursed by the DRGs system is greater than FFS system in Korea. These differences may result in respective price adjustments for individual diseases and insurance benefit approval for non-insured diagnoses. The Korean Hospital Association has contracted with insurers following the mandatory participation of DRGs, and many medical departments have complained strongly that such contracts were finalized without their agreement. In addition, they resisted the DRGs system until insurers reorganized the disease classification system and provided sufficient increases in reimbursements for patient care. As a result, insurers have adjusted their payment rates to encourage medical community participation in and to minimize discontent with the mandatory participation of the DRGs system during the second intervention period. However, we suggest that these price increases may be an inappropriate longterm policy. The government should investigate the original prices for medical practice, materials for medical treatment, and pharmaceuticals, and implement a revised plan with reasonable indemnification. Also, the proportion of un-insured patient costs that Korean hospitals can coordinate arbitrarily, compared to the total medical costs, is high. The large proportion causes a relatively low public health care expenditure of $56 \%$ in 2013, which was lower compared to the OECD average (72\%) and is the fourth lowest of OECD countries following Mexico (51\%), the United States (48\%), and Chile (46\%) [32]. The government has converted some uninsured expenses to insured costs, and insurers predict decreasing out-of-pocket costs by about $20 \%$ with the DRGs system. Therefore, potential increases in total medical expenses will be affected by these conversions of un-insured expenses. We suggest that the government need to examine alternations for stabilizing the application of un-insured costs by expanding the diseases and diagnoses included in the DRGs system.

Third, because the DRGs system reimburses costs for specific diseases based on a fixed price, physicians might shift inpatients to an outpatient service to decrease medical treatment or examination of inpatients. A prior report reported that after participation of the DRG system, the volume for pre-surgery examinations increased prior to hospitalization [33]. However, this study found no evidence that shifting services to outpatient settings increased following mandatory participation of the DRGs 
Table 3 Regression model estimates for shifting services to outpatient settings and readmission by DRG policy intervention

\begin{tabular}{|c|c|c|c|c|c|c|c|}
\hline \multirow[t]{3}{*}{ Variables } & \multirow{3}{*}{$\begin{array}{l}\text { Pre-Intervention } \\
7 / 2011-6 / 2012 \\
N(\%)\end{array}$} & \multicolumn{3}{|c|}{ First Intervention } & \multicolumn{3}{|c|}{ Second Intervention } \\
\hline & & \multicolumn{3}{|l|}{$7 / 2012-6 / 2013$} & \multicolumn{3}{|l|}{ 7/2013 6/2014 } \\
\hline & & N (\%) & $B$ & DID & N (\%) & $B$ & DID \\
\hline \multicolumn{8}{|c|}{$\begin{array}{l}\text { Shifting services to } \\
\text { outpatient settings }\end{array}$} \\
\hline \multicolumn{8}{|c|}{ Large hospital } \\
\hline $\mathrm{MPH}$ & $55,388(31.12)$ & $64,398(35.43)$ & $1.25^{* * *}$ & $0.94^{* * *}$ & $68,360(37.50)$ & $1.09^{* * *}$ & 1.00 \\
\hline VPH & $13,373(28.67)$ & $14,111(33.84)$ & $1.33^{* * *}$ & & $14,247(36.08)$ & $1.10^{* * *}$ & \\
\hline \multicolumn{8}{|c|}{ Small hospital } \\
\hline $\mathrm{MPH}$ & $12,141(14.29)$ & $20,047(18.46)$ & $1.13^{* * *}$ & 0.99 & $31,553(22.31)$ & $1.08^{* * *}$ & 1.02 \\
\hline VPH & $339,579(48.32)$ & $337,344(49.42)$ & $1.05^{* * *}$ & & $321,412(49.61)$ & $0.99^{*}$ & \\
\hline \multicolumn{8}{|c|}{ Readmission } \\
\hline \multicolumn{8}{|c|}{ Large hospital } \\
\hline $\mathrm{MPH}$ & $4,142(2.98)$ & $4,022(2.85)$ & $0.95^{*}$ & $1.14^{*}$ & $2,312(1.67)$ & $0.59^{* * *}$ & $0.82^{*}$ \\
\hline $\mathrm{VPH}$ & $597(1.42)$ & 433(1.16) & $0.85^{*}$ & & $300(0.85)$ & $0.73^{* * *}$ & \\
\hline \multicolumn{8}{|c|}{ Small hospital } \\
\hline $\mathrm{MPH}$ & $1,576(1.86)$ & $702(0.72)$ & $0.38^{* * *}$ & $0.40^{* * *}$ & $735(0.66)$ & 0.92 & 0.99 \\
\hline VPH & $3,103(0.86)$ & $2,829(0.82)$ & 0.95 & & $2,548(0.80)$ & 0.96 & \\
\hline
\end{tabular}

MPH mandatory participation hospitals

VPH voluntary participation hospitals

DID difference-in-differences

Large hospital: tertiary hospitals + general hospitals; Small hospital: hospitals+ clinics.

Adjusted odds ratios obtained from generalized estimating equations analysis with all of the variables in Table 1.

Patients with cataract surgery as the primary diagnosis were excluded in total analysis.

${ }^{*} p<0.05{ }^{* * *} p<0.001$

system. Although shifting services to outpatient settings for MPH patients increased significantly, a similar observed increase in VPH patients shifting services to outpatient settings suggests that mandatory DRGs participation is not the main cause of these transfers. A previous study that indicated the average number of medical tests conducted prior to admission increased from 3.51 to 4.46 following DRGs participation [28], although we attribute this difference to study design. Before controlling for other factors, we found that the rate of shifting services to outpatient settings increased by approximately 2-4 p.p. at MPHs, which corroborates the above-mentioned study. However, we considered a control group in our study design and believe this approach resulted in a more robust analysis of DRGs policy effects.

Finally, readmission rates, excluding those of patients with cataract surgery, decreased significantly after mandatory implementation of DRGs, indicating an overall improvement in the quality of patient care [34-44]. Our finding differed from previous research in which hospitals discharging patients early had increased readmission rates [45]. This study implied that DRGs payment systems may decrease quality of care because of "under-treatment" of specific conditions. But, there was no evidence that readmission rates decreased following mandatory participation of DRGs. These unexpected, yet positive, results may be due to more accurate medical coding, as insurers instruct all hospitals to submit separate DRGs claims, regardless of FFS claims already made. Our findings related to reduced readmission rates during the second intervention period supports this conclusion. Future research is warranted explore readmission rates of complicated procedures because the seven diseases applied by DRGs systems are treated by relatively simple surgical procedures.

This study has the a few limitations. First, our study did not consider un-insured costs in the total medical expenses. The Korean un-insured costs accounted for $24 \%$ of total medical expenditure in 2014. This research also did not explore alterations in out-of-pocket costs in the DRGs system because of our limited dataset. Second, we controlled for hospital characteristics in our regression models, although additional differences between MPHs and VPHs may have influenced our findings. For example, VPHs may have elected to participate in the DRG system because they already operated more efficiently than other hospitals, which could impact results on patient care independently of DRG implementation. Third, because we examined seven diseases, these study findings cannot be generalized to all diseases. Fourth, because our research has utilized the administrative data, 
our study might have overlooked any complications of the patients. But, we utilized CCI scores of each patient for evaluating the clinical characteristics of patients to resolve this limitation partially. Finally, there is the possibility that patients were first treated in a hospital under FFS and then in a hospital under the DRG system.

\section{Conclusions}

The mandatory participation of the DRGs system has caused significant decreases in LOS and readmission rate without increased shifting services to outpatient settings for patients with one of seven DRG-related diagnoses. Our findings support the continued implementation and enlargement of the DRGs payment system for other diseases in South Korea, given its potential for curbing unnecessary resource usage encouraged by FFS. If the government deliberates on expansion of the DRG system to include other diseases with higher rates of complications, policymakers need to monitor deterioration of health care quality caused by fixed pricing.

\section{Supplementary information}

Supplementary information accompanies this paper at https://doi.org/10. 1186/s12913-019-4650-8.

Additional file 1: Figure S1. Parallel trend assumption in case of large hospital. Figure S2. Parallel trend assumption in case of small hospital.

Additional file 2: Table S1. Regression model estimates for length-ofstay according to DRG policy intervention period and primary diagnosis. Table S2. Regression model estimates for total medical cost according to DRG policy intervention period and primary diagnosis. Table S3. Regression model estimates for shifting services to outpatient settings according to DRG policy intervention and primary diagnosis. Table S4. Regression model estimates for readmission according to DRG policy intervention and primary diagnosis.

\section{Abbreviations}

DRG: Diagnosis-related groups; VPH: Voluntary Participation Hospitals; MPH: Mandatory Participation Hospitals; FFS: Fee-For-Service; GEE: Generalized estimating equations; LOS: Length-Of-Stay; HIRA: Health Insurance Review and Assessment; OECD: Organization for Economic Cooperation and Development

\section{Acknowledgements}

The authors thank the Health Insurance Review and Assessment Service of Korea, which provide data from its KNHI Database.

\section{Authors' contributions}

JW contributed to study design and performed statistical analysis. SJ and HK reviewed previous literatures. TH and SI supported interpretations of the results. EC was responsible for drafting the manuscript. All authors approved the final version of the manuscript.

\section{Funding}

This study received no certain grant from any funding agency in the public, commercial, or not-for-profit sectors.

\section{Availability of data and materials}

The datasets generated and/or analysed during the current study are not publicly available because the Health Insurance Review and Assessment (HIRA) of Korea requests all related researchers to pledge not to review, share, or release the database. It is possible for other researchers to require access to the dataset directly from the HIRA.

\section{Ethics approval and consent to participate}

This study was exempted by ethical approval from the institutional review board of Yonsei University Graduate School of Public Health. The authors obtained all essential permission from the Health Insurance Review and Assessment Service (HIRA) to access the data before performing this study. Information for each patient was de-identified and anonymized by the HIRA.

\section{Consent for publication}

Not applicable

\section{Competing interests}

None of the authors has declared any conflicts of interest associated with this study.

\section{Author details}

${ }^{1}$ College of Pharmacy, Yonsei Institute of Pharmaceutical Sciences, Yonsei University, Incheon, Korea. ${ }^{2}$ Institute of Health Services Research, Yonsei University College of Medicine, 50-1 Yonsei-ro, Seodaemun-gu, Seoul, Korea. ${ }^{3}$ Department of Nursing, College of Nursing, Eulji University, Seongnam, South Korea. ${ }^{4}$ Department of International Cooperation, Health Insurance Review \& Assessment Service, Wonju, Korea. ${ }^{5}$ Department of Preventive Medicine, Yonsei University College of Medicine, 50-1 Yonsei-ro, Seodaemun-gu, Seoul, Korea. ${ }^{6}$ Department of Hospital Administration, Graduate School of Public Health, Yonsei University, Seoul, Korea.

Received: 26 August 2018 Accepted: 16 October 2019

Published online: 30 October 2019

\section{References}

1. Jung YW, et al. The Effect of Diagnosis-Related Group Payment System on Quality of Care in the Field of Obstetrics and Gynecology among Korean Tertiary Hospitals. Yonsei Med J. 2018;59(4):539-45.

2. Cheng SH, Chen CC, Tsai SL. The impacts of DRG-based payments on health care provider behaviors under a universal coverage system: a populationbased study. Health Policy. 2012;107(2-3):202-8.

3. Feinglass J, Holloway JJ. The initial impact of the Medicare prospective payment system on U.S. health care: a review of the literature. Med Care Rev. 1991;48(1):91-115.

4. Hill NT, Johns EL. Adoption of costing systems by U.S. hospitals. Hosp Health Serv Adm. 1994;39(4):521-37.

5. Busse R, Geissler A, Quentin W. Diagnosis-Related Groups in Europe. In: Moving Towards Transparency, Efficiency and Quality in Hospitals. New York: McGraw-Hill Education; 2011.

6. Jang Sl, et al. Impact of payment system change from per-case to per-diem on high severity patient's length of stay. Medicine (Baltimore). 2016;95(37): e4839.

7. Kim KH, et al. Does Korea's current diagnosis-related group-based reimbursement system appropriately classify appendectomy patients? Ann Surg Treat Res. 2016;91(2):66-73.

8. Lee K, Lee S. Effects of the DRG-based prospective payment system operated by the voluntarily participating providers on the cesarean section rates in Korea. Health Policy. 2007;81(2-3):300-8.

9. Kim $\mathrm{TH}$, et al. Effects of diagnosis-related group payment system on appendectomy outcomes. J Surg Res. 2016;206(2):347-54.

10. Shin SC, Kang KW, Kim SW. Simulation on the Change of Practice Pattern after the Introduction of 7 Diagnosis-related Groups Prospective Payment System in a University Hospital. Health Policy and Management. 2013;23(2): 103-11.

11. Kim Y, Seo JA, Jeong Y. Comparative Analysis of Medical Treatment Practices and Medical Costs between DRG Payment System and Fee For Service System. Medical Journal of Chosun University. 2002;27(1):166-76.

12. Lee ML, Lee YH, Ko KW. The Change of Medical Care Pattern and Cost of Cataract Surgery by the DRG Payment System in a General Hospital. Korean J Hosp Manag. 2005;10(1):48-70.

13. Kim HS, Shin JW, Woo JS. Patient Cost Analysis in one general hospital for an enhancement of the Korean Diagnosis Related Group (KDRG) system. Korean J Health Econ Policy. 2014;20(2):67-89.

14. Choi SJ, et al. Variation in hospital length of stay according to the DRGbased prospective payment system in the voluntarily participating providers. Health Policy Manag. 2010;20(2):17-39. 
15. Kim J, Yim E, Bae H. Performance of Drg-Based Reimbursement Policy In National Health Insurance : Eight Years' Experiences. Value Health. 2015;18(7):A546.

16. Kim SJ, et al. The effect of competition on the relationship between the introduction of the DRG system and quality of care in Korea. Eur J Public Health. 2015.

17. Kim L, Kim JA, Kim S. A guide for the utilization of Health Insurance Review and Assessment Service National Patient Samples. Epidemiol Health. 2014; 36:e2014008.

18. Hong JS, Kang HC, Kim J. Continuity of care for elderly patients with diabetes mellitus, hypertension, asthma, and chronic obstructive pulmonary disease in Korea. J Korean Med Sci. 2010;25(9):1259-71.

19. Hong JS, Kang HC. Relationship between continuity of ambulatory care and medication adherence in adult patients with type 2 diabetes in Korea: a longitudinal analysis. Med Care. 2014;52(5):446-53.

20. Kim H, et al. Early outcome of the Korean Diagnosis-Related Groups payment system for appendectomy. Ann Surg Treat Res. 2015;88(3):126-32.

21. Kwak SH, et al. Impact of the Korean Diagnosis-Related Groups payment system on the outcomes of adenotonsillectomy: A single center experience. Auris Nasus Larynx. 2018:45(3):504-7.

22. Shon C, et al. Impact of DRG payment on the length of stay and the number of outpatient visits after discharge for caesarean section during 2004-2007. J Prev Med Public Health. 2011;44(1):48-55.

23. Kim SJ, et al. Early Impact on Outpatients of Mandatory Adoption of the Diagnosis-Related Group-Based Reimbursement System in Korea on Use of Outpatient Care: Differences in Medical Utilization and Presurgery Examination. Health Serv Res. 2017.

24. Goldfield NI, et al. Identifying potentially preventable readmissions. Health Care Financ Rev. 2008;30(1):75-91.

25. Charlson ME, et al. A new method of classifying prognostic comorbidity in longitudinal studies: development and validation. J Chronic Dis. 1987;40(5):373-83.

26. Winkelmann R. Co-payments for prescription drugs and the demand for doctor visits--evidence from a natural experiment. Health Econ. 2004;13(11): 1081-9.

27. Chou SY, et al. Parental Education and Child Health: Evidence from a Natural Experiment in Taiwan. Am Econ J Appl Econ. 2010;2(1):63-91.

28. Kwon S. Payment system reform for health care providers in Korea. Health Policy Plan. 2003;18(1):84-92.

29. Wenke $A$, et al. Development of lengths of stay and DRG cost weights in dermatology from 2003 to 2006. J Dtsch Dermatol Ges. 2009;7(8):680-7.

30. OECD, Health at a Glance: Average length of stay in hospitals. 2015: OECD Publishing.

31. Rogers $W H$, et al. Quality of care before and after implementation of the DRG-based prospective payment system. A summary of effects. JAMA. 1990; 264(15):1989-94.

32. Choi JW, et al. Health care utilization and costs among medical-aid enrollees, the poor not enrolled in medical-aid, and the near poor in South Korea. Int J Equity Health. 2015;14(1):128.

33. Kim SJ, et al. Early Impact on Outpatients of Mandatory Adoption of the Diagnosis-Related Group-Based Reimbursement System in Korea on Use of Outpatient Care: Differences in Medical Utilization and Presurgery Examination. Health Serv Res. 2018;53(4):2064-83.

34. Dimick JB, Ghaferi AA. Hospital readmission as a quality measure in surgery. JAMA. 2015:313(5):512-3.

35. Tapper EB, et al. A Quality Improvement Initiative Reduces 30-Day Rate of Readmission for Patients With Cirrhosis. Clin Gastroenterol Hepatol. 2015.

36. Khan A, et al. Same-Hospital Readmission Rates as a Measure of Pediatric Quality of Care. JAMA Pediatr. 2015;169(10):905-12.

37. Fischer $C$, et al. A systematic review and meta-analysis on the association between quality of hospital care and readmission rates in patients with heart failure. Am Heart J. 2015;170(5):1005-17 e2.

38. Wish JB. The role of 30-day readmission as a measure of quality. Clin J Am Soc Nephrol. 2014;9(3):440-2

39. Girotti ME, Shih T, Dimick JB. Health policy update: rethinking hospital readmission as a surgical quality measure. JAMA Surg. 2014;149(8):757-8.

40. Fischer $C$, et al. Is the readmission rate a valid quality indicator? A review of the evidence. PLoS One. 2014:9(11):e112282.

41. Cohn A. Readmission rates are a poor marker of quality. BMJ. 2014;348: g1148.

42. Chen Q, et al. Using a composite readmission measure to assess surgical quality in the Veterans Health Administration: how well does it correlate with established surgical measures? JAMA Surg. 2014;149(11):1206-7.
43. Tsai TC, et al. Variation in surgical-readmission rates and quality of hospital care. N Engl J Med. 2013:369(12):1134-42.

44. Jian W, et al. Payment Reform Pilot In Beijing Hospitals Reduced Expenditures And Out-Of-Pocket Payments Per Admission. Health Aff (Millwood). 2015;34(10):1745-52.

45. Hendren S, et al. Early discharge and hospital readmission after colectomy for cancer. Dis Colon Rectum. 2011:54(11):1362-7.

\section{Publisher's Note}

Springer Nature remains neutral with regard to jurisdictional claims in published maps and institutional affiliations.
Ready to submit your research? Choose BMC and benefit from:

- fast, convenient online submission

- thorough peer review by experienced researchers in your field

- rapid publication on acceptance

- support for research data, including large and complex data types

- gold Open Access which fosters wider collaboration and increased citations

- maximum visibility for your research: over $100 \mathrm{M}$ website views per year

At BMC, research is always in progress.

Learn more biomedcentral.com/submissions 